\title{
Lemierre Syndrome Complicating Pharyngotonsilitis in a Young Male: A Case Report
}

\author{
Segun Patrick Adeoye ${ }^{\mathrm{a}, \mathrm{b}, \mathrm{f}}$, Sherly Abrahamc, Irina Erlikh ${ }^{\mathrm{c}}$, Idelka Rodriguez-Rosaric ${ }^{\mathrm{c}}$, \\ Jean Itaney Toussaint ${ }^{\mathrm{c}}$, Sylvester Sarfraz ${ }^{\mathrm{c}, \mathrm{d}}$, Tomas Borda ${ }^{\mathrm{e}}$
}

\begin{abstract}
Lemierre syndrome is a very rare complication of head and neck infections. We present a case report on a healthy young male who developed unremitting fever, marked leucocytosis, multiple pulmonary consolidation, and internal jugular vein thrombosis in the setting of a pharyngotonsilitis. The atypicality of the presentation is emphasized, diagnostic and therapeutic considerations and complications are discussed. This report makes the case for entertaining a diagnosis of Lemierre' syndrome complicating antecedent head and neck space infection especially when presentation is atypical or response to conventional care is suboptimal.
\end{abstract}

Keywords: Lemierre' syndrome; Pharyngotonsilitis; Internal jugular vein thrombosis

\section{Introduction}

Lemierre syndrome, notoriously described as the "forgotten or neglected disease" $[1,2]$ is a rare thrombophlebitic complication of common head and neck infections. It is more commonly reported in healthy young adults with a reported incidence of less than 1 per million of the population $[1,3]$. In $87 \%$ of cases, there is an antecedent pharyngotonsilitis, with subsequent Fusobactrium necrophorum invasion of the para and retropharyngeal spaces $[4,5]$. Other microbes

\footnotetext{
Manuscript accepted for publication August 6, 2013

${ }^{a}$ University of Pittsburgh Medical Center (Horizon), PA, USA

${ }^{b}$ Formerly of Department of Family Medicine, The Brooklyn Hospital Center, NY, USA

${ }^{\mathrm{c}}$ Department of Family Medicine, The Brooklyn Hospital Center, NY, USA

${ }^{\mathrm{d}}$ Department of Geriatric Medicine, Brown University/Rhode Island Hospital, Providence, RI, USA

${ }^{\mathrm{e}}$ Weil Cornell University/Hospital, NY, USA

${ }^{\mathrm{f}}$ Corresponding author: Segun Patrick Adeoye, University of Pittsburgh Medical Center (Horizon), 110 N Main Street, Greenville, PA 16125,

USA. Email: adeoyesp@upmc.edu
}

doi: http://dx.doi.org/10.4021/jmc1448w implicated in the aetiopathogenesis of Lemierre's include anaerobes and gram negatives like other Fusobacterium species, Bacteroides fragilis, Bacteroides melaninogenicus, Peptostreptococcus spp, Porphyromonas asaccharolytica and Eikenella corrodens [2, 6, 7]. Ebstein-Barr Virus has also been implicated but its role is less clearly understood; some authors suggesting infectious mononucleosis may rarely precede Lemierre syndrome [8]. Lemierre syndrome may also complicate otitis media, mastoiditis, sinusitis and parotitis. There are reports of Staphylococcus catheter sepsis related Lemierre syndrome. The usual course is the pervasion by Fusobacterium species of the streptococcal-infected pharyngotonsilar epithelium to access the anaerobic environment of the deep pharyngotonsilar planes. While thriving under these abetting conditions, Fusobacterium invades subserving veins and carotid sheath, leading to an initial internal jugular vein (IJV) thrombophlebitis/thrombosis and ultimately, embolic phenomena with septic foci to distant sites [2]. Symptomatology is constitutional or related to the affected organs and/ or systems, fever and gastrointestinal symptoms present in $82 \%$ and $50 \%$ of cases respectively [9]. The thromboembolic complication may include one or various combinations of the following: bacteremia, pneumonia; pericarditis; septic foci in muscles; liver, spleen (with hepatosplenomegaly); kidney and intracranial abscesses; and meningitis. Cranial nerve palsies, Horner's syndrome, trismus, and muscle paralysis have been reported in cases with leptomeningitic complications [10]. Septic embolus to the lung is the most common embolic complication occurring in over $95 \%$ of cases [11]. Moreno and Altozano in Madrid reviewed cases of Lemierre's syndrome with pulmonary septic emboli [12]. They report that features relatable to assault on the lungs usually manifest early (even on the first day of the septicemia); symptoms include cough (unusual for streptococcal pharyngitis), pleuritic chest pain and hemoptysis [9]. Differential diagnosis may include tuberculosis, pneumonia, and Q-fever. Treatment usually involves antibiotics (parentally, initially) with or without anticoagulation. General and specific hematological, biochemical, and microbiological studies are immensely helpful in the diagnostic work-up. Computed tomography of neck with intravenous contrast is the most definitive study to confirm IJV thrombosis. MRI of the 

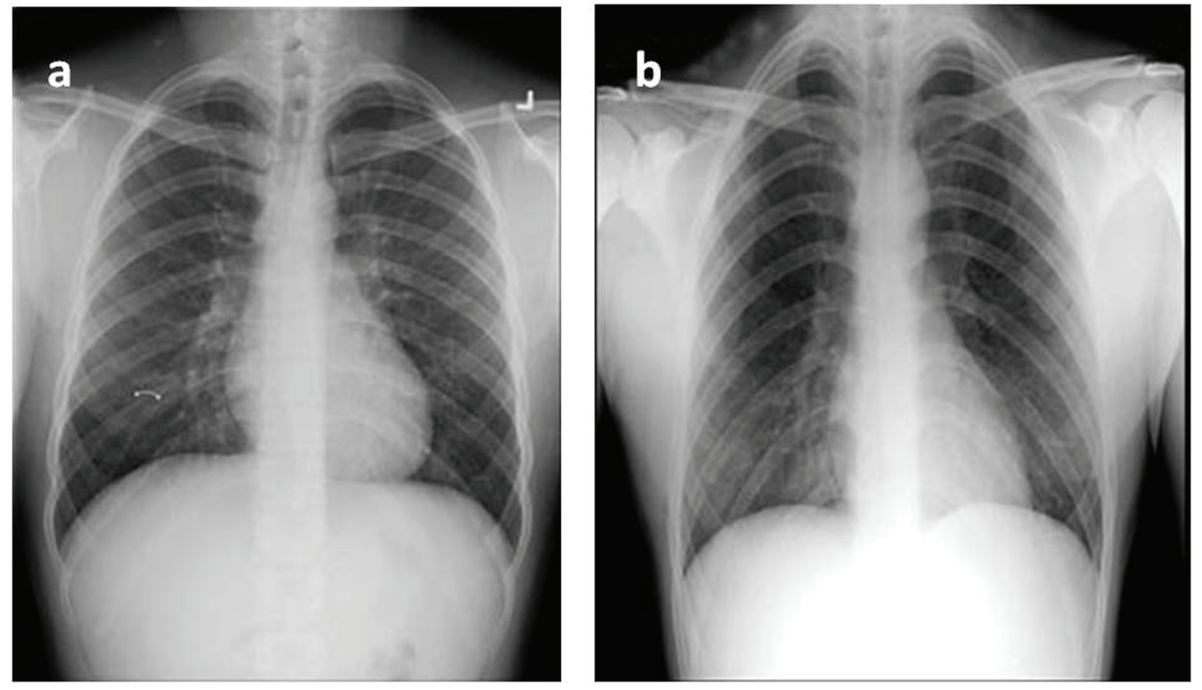

Figure 1. Chest radiography at admission (a) and on day 6(b): see appearance of opacities.

neck has been used but it not superior to $\mathrm{CT}$ and in practice is not always readily available. Neck ultrasonography is less sensitive. Chest radiography, and when indicated, contrast tomography of the chest will reveal pulmonary septic embolic pulmonary events. Sonography of abdomen may reveal abdominal pathology. Treatment involves antibiotics (parentally, initially) with or without anticoagulation, as well as supportive care [13]. Recommended antibiotic regimen is a beta-lactam resistant antibiotic, ampicillin-sulbactam, piperacillin-tazobactam ticarcillin-clavulanate [11]. Carbapenem and clindamycin monotherapy have been tried with success in some cases [4]. Vancomycin is advocated for catheter sepsis associated IJV thrombophlebitis. There are reports of patients requiring drainage of forming abscesses and surgical ligation or excision of the internal jugular vein for recalcitrant cases $[4,14,15]$. Disseminated disease is associated with more complications, longer hospital stay and higher mortality [4]. Mortality for properly treated Lemierre's estimated at $4.6-22 \%[4,16]$.

\section{Case Report}

JD, a 27-year-old male patient presented with a 4-day history of soreness in the throat and with subjective high-grade fevers, chills, nausea, and vomiting. Vomitus was non-projectile, non-billious, and contained recently ingested meals and mucous. He complained of odynophagia and neck pain rated as 10/10 in severity, maximum in the left submandibular area with radiation to his left mandible and left temporal area. Neck pain was aggravated by neck movements and partially relieved by acetaminophen. JD lives with his girl friend who recently recovered from sore throat. Her course was uneventful.
JD's medical history was significant for appendicectomy 14 years ago. He reported no allergies, drinks alcohol socially, with 5-pack-year cigarette smoking history. He has no recent and past use of illicit drugs. JD works for an information technology firm while pursuing a business degree at a local college. He is sexually active with a steady girl friend of many years, sexual encounters are often unprotected. Family history was unremarkable.

Relevant negatives noted on system review included the absence of cough and chest pain.

On physical exam, the patient was ill looking but not in any form of distress. He was febrile $(102.1 \mathrm{~F})$, tachycardic (rate: 119 beats / min). He had herpetic blisters on his lips and angle of mouth, florid dental plaques with periodontal lesions. Bilateral tonsilar hypertrophy with injection and retrotonsilar exudates were observed. He was tender in the left jugulo-digastric area, and resisted deep palpation. Chest and cardiovascular examination was essentially normal (besides observed tachycardia). He was vaguely tender in the epigastrium, no hepatomagaly was appreciated, Murphys's was equivocal, and spleen was tipped on palpation. Neurological and musculoskeletal examination was normal.

A Centor score of $4 / 4$ and positive bedside rapid streptococcal was documented. Abnormalities noted on CBC were isolated neutrophilia of $79 \%$ (without leucocytosis) and thrombocytopenia $(89,000)$. Liver function test (LFTs) reported elevated total and direct bilirubin (3.3 and 2.2 respectively), with mild elevation of AST(42 U/L) and ALP(218 $\mathrm{U} / \mathrm{L})$. C-reactive protein was markedly elevated $(159 \mathrm{mg} / \mathrm{L})$. Basic metabolic panel, amylase and lipase were within normal limits. Heterophile antibody test and hepatitis profile were negative (patient was immune to hepatitis B, evident in a positive $\mathrm{HBsAb}$ ). Chest $\mathrm{X}$-ray done at admission was unremarkable (Fig. 1a). 

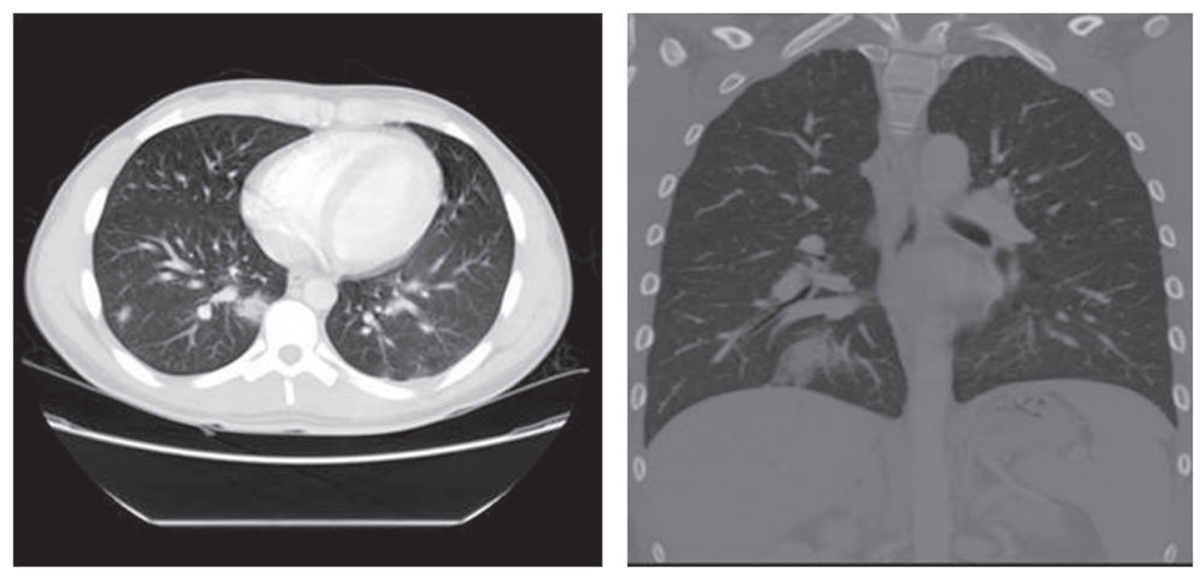

Figure 2. Chest CT with contrast done on day 4: see embolic foci and consolidations [22].

After care conferencing between the emergency room and the admitting teams, a decision was made to admit the patient, as the patient was toxic looking and tolerance for oral antibiotics was doubtful. Initial management was instituted with intravenous hydration, antibiotics and analgesia. In view of the mild splenomegaly, and absent definitive exclusion of infectious mononucleosis, azithromycin was selected as initial antibiotic of choice.

After initial clinical improvement, patient condition deteriorated with increasing fevers, headaches, and neck pain, and interval leucocytosis. Initial throat culture and panculture yielded no organisms. A decision to explore for a probable complicating peritonsillar abscess and acute cholecystitis was made; an infectious disease consult was obtained on the third day of admission. An ultrasound of the right upper quadrant reported gall bladder wall thickening, periportal edema, and splenomegaly without cholelithiasis or cholelithiasis. A subsequent HIDA scan was consistent with the finding on ultrasound. Repeat chest X-ray showed bilateral infiltrates (Fig. 1b), a finding later confirmed by chest CT showing multiple bilateral pulmonary consolidations (Fig. 2). An ultrasound study of the neck was ordered to evaluate for peritonsillar abscess. The neck sonogram revealed soft tissue swelling, extensive lymphadenopathy and thrombosis of left IJV. In view of its diagnostic superiority over ultrasound in evaluating vasculature deep to clavicle and mandible, neck CT with intravenous contrast was ordered to fully characterize the nature and extent of the thrombus [4]. Its finding was consistent, confirming extensive lymphadenopathy and a $5 \mathrm{~cm}$ thrombus in the left IJV. At this point, a diagnosis of IJV thrombophlebitis with thrombosis, septic embolism and pneumonia was made, all consistent were with Lemierre' syndrome.

PPD and HIV testing performed to exclude tuberculosis and acquired immunosuppression reported negative. Antibiotic therapy was reviewed, consistent with antibiotic recommendation for Lemierre syndrome, azithromycin was held, vancomycin and unasyn (ampicillin plus sulbactam) started. After a thoughtful risk-benefit analysis, a decision was made to commence anticoagulation. The decision was informed by recent deterioration in the patient's clinical status as well as the consideration of the potential for thrombus extension and further embolic events. Patient received warfarin therapy with initial enoxaparin bridging. Patient made remarkable clinical improvement, evident in improved resolution of fever and pain, as well normalization of hematotological and biochemical indices. However, AST, ALT, and ALP values increased, suggesting new hepatobiliary insult, ampicillinsulbactam was identified as the likely culprit. Upon reconsulting the infectious disease specialist, a combination therapy of ceftriaxone/metronidazole was recommended as it provided potentially equivalent antimicrobial efficacy as vancomycin/unasyn therapy without adverse hepatobiliary profile. JD received two weeks of intravenous antibiotic therapy, making complete clinical recovery. He was scheduled to complete two further weeks of oral antibiotic therapy and complete 13 weeks anticoagulation therapy. He was discharged with a detailed plan for outpatient follow-up under care of his primary care physician.

\section{Discussion}

In the pre-antibiotic era Lemierre's Syndrome was a common complication of severe oropharyngeal infection [9] with an associated mortality rate over $90 \%$ [17]. In the modern antiboitic era, Lemierre' syndrome complicating pharyngotonsilitis very seldom occurs. Eykin et al reported that they could not find a physician who had heard of the disease [18]. Chirinos et al (2002) asserted that a high degree of clinical suspicion is necessary for diagnosis [4]. The decision to admit JD was informed by his toxic presentation. Retrospectively, the decision was a good call. We make following postulations: 
1). The precipitating event was an antecedent streptococcal pharyngotonsilitis. This assertion is supported by the presence of fever, absence of cough, bilateral tonsilar exudate, tender jugular digastric lymphadenopathy (4/4 on Centor scale), and a positive rapid streptococcal test. A negative throat culture is not inconsistent with a diagnosis of streptococcal pharyngotonsilitis. Culture testing is $75-90 \%$ sensitive and $95-99 \%$ specific $[19,20]$. False negative may be explained by sub-optimal specimen collection, poor laboratory processing, and as well recent antibiotic exposure [4].

2 ). The patient's poor dental status and observed periodontal disease was complementary: a supportive for the oral anaerobes to flourish. Furthermore, the anaerobic environment created by the cellulitic parapharyngotonsilar tissues, bolster growth of Fusobacterium species. Routine throat and blood culture have poor yield for anaerobes because of the requirement for anaerobic melieu for optimal growth.

3). The observed thrombocytopenia is explained by Fusobacterium hemaglutinin platelet aggregation. Thrombocytosis have been reported in some cases of Lemierre's.

4). The initial elevation in AST and ALP, as well as finding of gall balder thickening (without cholelithiasis) and periportal edema is explained by some form of acalculous cholecystitis related to microveno-occlusive events from sepsis or septic thromboembolism.

5). The worsening neck pain and radiating headaches was a harbinger of on-going septic thrombophlebitis and IJV thrombosis.

6). The interval development of multiple pulmonary consolidations confirmed on subsequent imaging is the result of septic embolism from the thrombosed IJV. Normal chest radiograph at admission with interval changes is not inconsistent with the course of Lemierre's Syndrome.

7). The worsening hepatic indices are explained by ampicillin-sulbactam-induced cholestatsis; the hypothesis is supported by the return to normalcy of these indices upon suspending ampicillin-sulbactam. Ampicillin-sulbactam induced cholestatis and hepatitis is not an uncommon clinical event.

8). The use of anticoagulation in Lemierre is controversial. The absence of controlled studies is explained by the rarity of the disease [15]. Current recommendation is based on consensus opinions, many experts advising against anticoagulation for non-extensive IJV thrombosis [12]. However, it was determined that, despite the non-extensive nature of the IJV thrombus in this case, the risks-benefit analysis favored anticoagulation as the disease was still evolving, with significant potential for thrombus extension and the propagation of embolic events.

\section{Conclusion}

Physicians should have a low threshold for entertaining a diagnosis of Lemierre' syndrome, amongst other differentials, in patients with head and neck infection with atypical presentation, or who fail to respond optimally to conventional antibiotic therapy. This case emphasizes the importance of team work and the exploitation of all diagnostic cues and consideration even with the simplest diagnoses and surety of outcomes. Atypicality of presentation or unusuality of disease course should not elicit a diagnosis of "malingering", (as was considered in this case), it is a recipe for disaster. In the light of the issues raised in this report, awareness of the disease should be promoted through physician education, medical reporting in scientific journals, as well as inclusion in test items in board certification examinations. Lemierre's syndrome should no longer be seen as a forgotten disease, rather as a re-emerging one.

\section{References}

1. Baker CC, Petersen SR, Sheldon GF. Septic phlebitis: a neglected disease. Am J Surg. 1979;138(1):97-103.

2. Screaton NJ, Ravenel JG, Lehner PJ, Heitzman ER, Flower CD. Lemierre syndrome: forgotten but not extinct--report of four cases. Radiology. 1999;213(2):369374.

3. Hagelskjaer Kristensen L, Prag J. Lemierre's syndrome and other disseminated Fusobacterium necrophorum infections in Denmark: a prospective epidemiological and clinical survey. Eur J Clin Microbiol Infect Dis. 2008;27(9):779-789.

4. Chirinos JA, Lichtstein DM, Garcia J, Tamariz LJ. The evolution of Lemierre syndrome: report of 2 cases and review of the literature. Medicine (Baltimore). 2002;81(6):458-465.

5. Wright WF, Shiner CN, Ribes JA. Lemierre syndrome. South Med J. 2012;105(5):283-288.

6. Morizono S, Enjoji M, Sonoda N, Fukushima M, Kuniyoshi M, Kotoh K, Nakamuta M, et al. Lemierre's syndrome: Porphyromonas asaccharolytica as a putative pathogen. Intern Med. 2005;44(4):350-353.

7. Sinave CP, Hardy GJ, Fardy PW. The Lemierre syndrome: suppurative thrombophlebitis of the internal jugular vein secondary to oropharyngeal infection. Medicine (Baltimore). 1989;68(2):85-94.

8. Riordan T, Wilson M. Lemierre's syndrome: more than a historical curiosa. Postgrad Med J. 2004;80(944):328334.

9. Lemierre A. On certain septicaemias due to anaerobic organisms. Lancet. 1936;1:701-703.

10. Peer Mohamed B, Carr L. Neurological complications in two children with Lemierre syndrome. Dev Med Child Neurol. 2010;52(8):779-781.

11. Golpe R, Marin B, Alonso M. Lemierre's syndrome (necrobacillosis). Postgrad Med J. 1999;75(881):141144. 
12. Moreno S, Garcia Altozano J, Pinilla B, Lopez JC, de Quiros B, Ortega A, Bouza E. Lemierre's disease: postanginal bacteremia and pulmonary involvement caused by Fusobacterium necrophorum. Rev Infect Dis. 1989;11(2):319-324.

13. Weeks DF, Katz DS, Saxon P, Kubal WS. Lemierre syndrome: report of five new cases and literature review. Emerg Radiol. 2010;17(4):323-328.

14. Spelman D, Sexton D, Baron E. Suppurative (septic) thrombophlebitis. HYPERLINK "http://www.uptodate. com" www.uptodate.com.

15. Puymirat E, Biais M, Camou F, Lefevre J, Guisset O, Gabinski C. A Lemierre syndrome variant caused by Staphylococcus aureus. Am J Emerg Med. 2008;26(3):380 e385-387.
16. Karkos PD, Asrani S, Karkos CD, Leong SC, Theochari EG, Alexopoulou TD, Assimakopoulos AD. Lemierre's syndrome: A systematic review. Laryngoscope. 2009;119(8):1552-1559.

17. Beldman TF, Teunisse HA, Schouten TJ. Septic arthritis of the hip by Fusobacterium necrophorum after tonsillectomy: a form of Lemierre syndrome? Eur J Pediatr. 1997;156(11):856-857.

18. Eykyn SJ. Necrobacillosis. Scand J Infect Dis Suppl. 1989;62:41-46.

19. Kellogg JA, Manzella JP. Detection of group A streptococci in the laboratory or physician's office. Culture vs antibody methods. JAMA. 1986;255(19):2638-2642.

20. Brien JH, Bass JW. Streptococcal pharyngitis: optimal site for throat culture. J Pediatr. 1985;106(5):781-783. 